\title{
Influencia del estrógeno en la enfermedad periodontal. Revisión de literatura
}

\author{
Influence of estrogen in disease periodontal. Literature Review
}

\author{
PAZMINO VFC* \\ ASSEM NZ* \\ PELLIZZER EP** \\ DE ALIMEIDA JIM*** \\ THEODORO LH***
}

\begin{abstract}
Pazmino VFC, Assem NZ, Pellizzer EP, De Almeida JM, Theodoro LH. Influencia del estrógeno en la enfermedad periodontal. Revisión de
\end{abstract} literatura. Av Periodon Implantol. 2015; 27, 2: 75-78.

\begin{abstract}
RESUIMEN
La etiología de la enfermedad periodontal está bien definida, dentro de los agentes etiológicos que la causan podemos citar algunos microorganismos subgingivales como: Porphyromonas gingivalis, Prevotella intermedia, Bacteroides forsythus, Actinobacillus actinomycetemcomitans y espiroquetas. La susceptibilidad del huésped a estos agentes bacterianos también tiene un papel importante dentro del progreso y prevalencia de la enfermedad periodontal. Dentro de los factores de riesgo asociados con la enfermedad periodontal tenemos: el aumento de edad, presencia de biofilm dental o placa bacteriana patogénica, alteración del estado inmunológico, deficiencia nutricional, el uso de medicamentos como corticoides, género, estrés, tabaco, factores genéticos y condiciones sistémicas, dentro de estas se incluyen alteraciones de neutrófilos, diabetes, embarazo, osteoporosis y hormonales. La enfermedad periodontal puede ser exacerbada por determinados factores sistémicos como la deficiencia o aumento de hormonas como estrógeno y progesterona. La presente revisión de literatura tiene por objeto dilucidar la influencia del estrógeno en la enfermedad periodontal.
\end{abstract}

PALABRAS CLAVE: Estrógeno, enfermedad periodontal, reabsorción ósea.

\section{SUMIMARY}

The etiology of periodontal disease is well defined within the etiologic agents that cause this disease. We can cite some subgingival microorganisms as gingivallis Porphyromonas, Prevotella intermedia, Bacteroides forsythus, Actinobacillus actinomycetemcomitans and spirochetes. The host susceptibility to these bacterial agents also has an important role in the progress and prevalence of periodontal disease. Among the risk factors associated with periodontal disease are: increasing age, presence of dental biofilm or pathogenic plaque, altered immune status, nutritional deficiency, use of medications such as corticosteroids, gender, stress, snuff, genetic factors and systemic conditions within these alterations are included neutrophils, diabetes, pregnancy, osteoporosis and hormones. Periodontal disease can be exacerbated by certain systemic factors such as deficiency or increased hormones like estrogen and progesterone. This literature review aims to elucidate the influence of estrogen in periodontal disease.

KEY WORDS: Estrogen, periodontal disease, bone resorption.

Fecha de recepción: 15 de enero de 2014.

Fecha de aceptación: 1 de mayo de 2014.

* $\quad$ Alumno de Maestría en Periodoncia.

** Profesor Adjunto. Doctor en Prótesis.

*** Profesor Asistente. Doctor en Periodoncia.

Universidade Estadual Paulista "Júlio de Mesquita Filho", Faculdade de Odontologia de Araçatuba, Departamento de Cirurgia

e Clínica Integrada - Disciplina Periodontia/UNESP. 


\section{INTRODUCCIÓN}

Las enfermedades periodontales están asociadas a bacterias patógenas específicas que colonizan el área subgingival. Su inicio y progresión son claramente modificadas por las condiciones sistémicas, denominadas factores de riesgo (1).

Las alteraciones en los niveles hormonales sexuales están ligadas más frecuentemente a las mujeres que a los hombres, esto se debe a que el estrógeno y la progesterona son predominantes en el sexo femenino. Estas hormonas parecen ejercer efectos más significativos y potencialmente más perjudiciales sobre el metabolismo de los tejidos, y a más de eso, las mujeres pasan por diferentes etapas fisiológicas que alteran estos niveles hormonales como menstruación, embarazo, menopausia, posmenopausia y el uso de anticonceptivos que son situaciones únicas del sexo femenino (2).

Ciertos factores modificadores hormonales pueden aumentar la prevalencia, incidencia y severidad de las gingivitis y periodontitis (3). Autores relataron que cambios en los niveles hormonales que ocurren durante la menopausia, pubertad, menstruación y embarazo, así como aquellos que suceden con el uso de suplementos hormonales, pueden llevar a una pérdida de la homeostasis del periodonto, facilitando el desenvolvimiento de la gingivitis (4).

En la presente revisión de literatura, se investiga la influencia del estrógeno en la enfermedad periodontal y la modificación del periodonto en las diferentes etapas de vida de la mujer, tales como: pubertad, menstruación, embarazo y menopausia.

\section{MATERIAL Y MÉTODOS}

Las palabras claves fueron: estrógeno, enfermedad periodontal y reabsorción ósea. Las bases de datos utilizadas fueron: PubMed/MEDLINE y Periodicos Capes. La investigación comprendió un período de tiempo de 10 años, desde junio de 1993 a junio del 2013. Dentro de los criterios de inclusión usados están: artículos, revisiones de literatura y casos clínicos sobre la influencia del estrógeno en las diferentes etapas de vida de la mujer (pubertad, menstruación, embarazo y menopausia). Fueron usadas publicaciones en idioma Inglés y Portugués. Para los criterios de exclusión descartamos trabajos realizados en animales, y que no fuesen publicados en inglés y portugués.

\section{RESULTADOS}

Fueron incluidas 9 publicaciones ( 7 en inglés y 2 en portugués), de las cuales, 4 fueron revisiones de literatura y 5, artículos científicos. Siendo que 6 de ellos evaluaron el nivel de estrógeno en las diferentes etapas de vida de la mujer como: pubertad, menstruación, embarazo, menopausia, el uso de contraceptivos orales, y se logró comprobar que había alteración en los niveles de esta hormona (exceso o disminución), produciendo efectos significativos en el tejido perio-

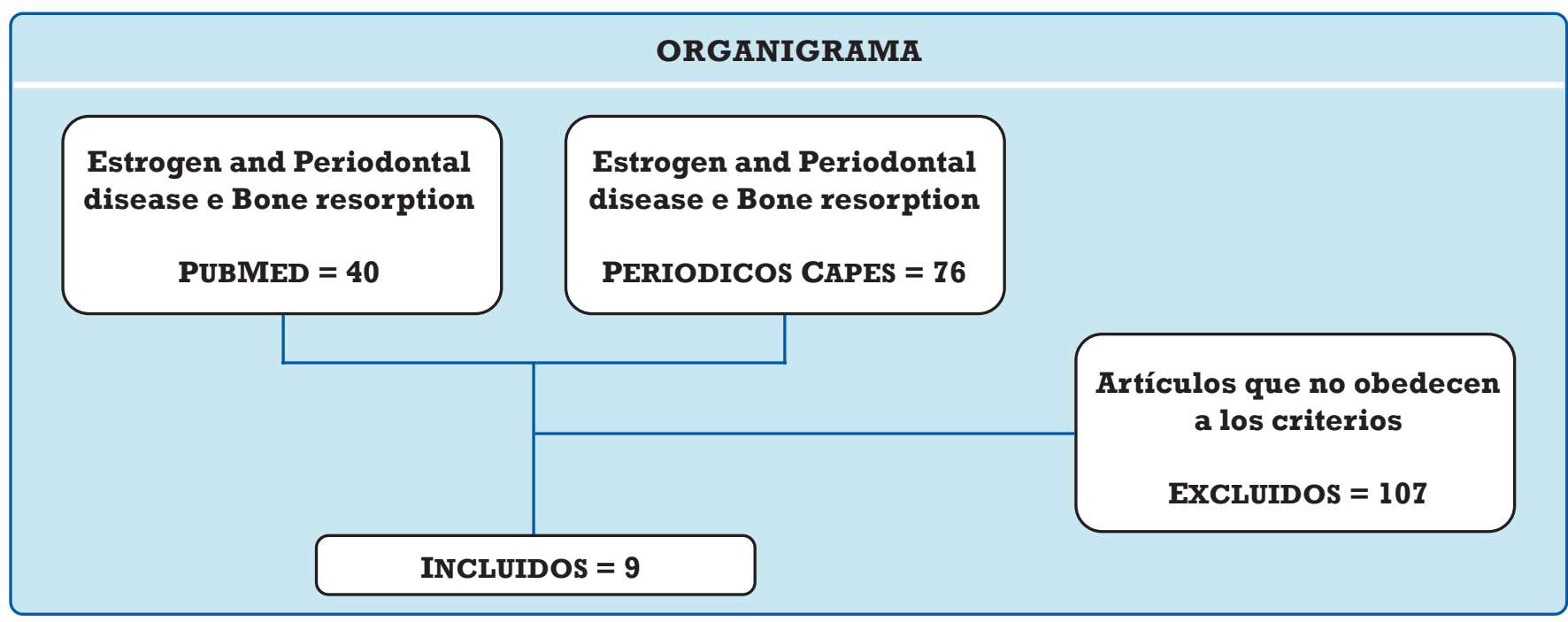

Fig. 1: Organigrama de la investigación de los artículos científicos. 
dontal. Los 3 estudios restantes analizaron también: movilidad, profundidad de sondaje, recesión gingival, inflamación gingival, nivel de inserción, en pacientes menopáusicas, con y sin terapia hormonal de reposición (THS), y los efectos del ciclo menstrual en la enfermedad periodontal.

Autores relataron los principales efectos que esta hormona causa sobre los tejidos periodontales y son (5-13):

- Provoca disminución de la queratinización y aumento del glucógeno epitelial que resulta en la reducción de la eficacia de la barrera epitelial.

- Aumenta la proliferación celular en la sangre.

- Estimula la fagocitosis de los Polimorfonucleares (PMNs).

- Inhibe la quimiotaxis de los PMNs.

- Suprime la producción de leucocitos del hueso medular.

- Inhibe las citosinas proinflamatorias liberadas por la médula humana.

- Reduce las células-T mediadores de la inflamación.

- Estimula la proliferación de los fibroblastos de la gingiva.

- Aumenta la cantidad de inflamación gingival sin aumento de placa.

- Estimula la síntesis de maduración del tejido conjuntivo gingival.

- Afecta a las peroxidasas salivales.

- Posee efecto estimulador sobre el metabolismo del colágeno y angiogénesis.

- Desencadena las vías autocrinas y paracrinas de señalización del factor de crecimiento polipeptídico.

\section{DISCUSIÓN}

Existe una gran propensión de pérdida ósea alveolar en pacientes pos menopáusicas con osteoporosis, especialmente cuando tienen enfermedad periodontal preexistente. Debido a la limitada cantidad de artículos existentes sobre este tema en los últimos 10 años, no está totalmente claro el papel del estrógeno con la osteoporosis en la enfermedad periodontal, por lo que son necesarios más estudios (5).

La deficiencia de estrógeno promueve pérdida de masa ósea en las mujeres menopáusicas (14), sin embargo, en un estudio en mujeres menopáusicas con TRH (Terapia de Reposición Hormonal) y sin TRH, demostraron que la influencia del estrógeno no tiene diferencias estadísticamente significativas en la condición periodontal de las pacientes evaluadas (12).
Revisando los efectos de la menopausia en los tejidos periodontales, se identificaron a ésta como un factor de riesgo para las enfermedades periodontales, sumada a factores socioeconómicos, demográficos, genéticos y sistémicos. Se evaluaron que a consecuencia del desequilibrio en la remodelación ósea que existe y por el aumento de las reacciones innatas de las respuestas inflamatorias como la Interleucina 1, IL-6 IL-8, IL-10, factor de necrosis tumoral, factor de estimulación de los granulocitos-macrófagos, modula la proliferación de células óseas e induce la reabsorción de tejido óseo esqueletal y alveolar (9); por otro lado, estudiando la TRH, en la condición periodontal en mujeres pos menopáusicas, demostraron que ésta, a largo plazo no está asociada con ningún efecto significativo sobre el estado periodontal y sobre las medidas clínicas de enfermedad periodontal de las pacientes examinadas (11).

En una revisión de literatura en la que evaluaron la influencia de las hormonas sexuales femeninas sobre las manifestaciones clínicas en las enfermedades periodontales, concluyeron que los niveles de estrógeno y progesterona poseen un efecto modulador sobre la respuesta inflamatoria en el huésped frente a las agresiones bacterianas (13). Las hormonas sexuales femeninas por sí mismas no son suficientes para producir alteraciones gingivales; sin embargo, pueden alterar las respuestas de los tejidos periodontales frente a la placa bacteriana e indirectamente contribuir en la enfermedad periodontal (8).

El estrógeno puede influenciar el estado del periodonto en la mujer en sus diferentes etapas de variación hormonal, tales como: la pubertad, menstruación, embarazo, menopausia y posmenopáusica (6).

La deficiencia de estrógeno promueve pérdida de masa ósea, en consecuencia el desequilibrio en la remodelación ósea, lo cual determina una mayor reabsorción en relación con la neoformación ósea (15). Por otro lado, mediante la terapia de reposición de estrógeno, la perdida de hueso alveolar propiamente dicha son prevenidas (16).

La mayoría de los autores son unánimes en decir que existe influencia de los niveles de estrógeno en los tejidos periodontales, asociados a las diversas etapas de la vida de la mujer, pero las opiniones son diversas cuando se refiere a la progresión de la pérdida de inserción clínica característica de las periodontitis. Son necesarios más estudios para obtener un mejor entendimiento de cómo esta hormona influencia la condi- 
ción periodontal sobre todo en los cambios hormonales existentes en mujeres.

\section{CONSIDERACIONES FINALES}

Está suficientemente comprobado en la literatura que cualquier cambio en los niveles de estrógeno, cuyo evento está asociado directamente a los cambios que sufre la mujer en sus diferentes etapas de vida, provoca un efecto que altera la respuesta inflamatoria del huésped frente a las agresiones bacterianas cuando existe enfermedad periodontal. A pesar de existir limitaciones y variaciones metodológicas en la cantidad de experimentos científicos que buscan investigar este hecho, la literatura comprueba solo modificaciones en la forma de manifestación clínica asociada a la gingivitis, sin alterar el desenvolvimiento de la perdida de inserción que caracteriza a las periodontitis.

\section{BIBLIOGRAFÍA}

1. Genco RJ. Current view of risk factors for periodontal diseases. J Periodontol 1996;67:1041-9.

2. Carranza FA Jr. Current state of the etiological problem of periodontal disease. Rev Bras Odontol 1970;27:269-75.

3. Kinane DF. Periodontitis modified by systemic factors. Ann Periodontol 1999;4:54-64.

4. Krejci CB, Bissada NF. Women's health issues and their relationship to periodontitis. J Am Dent Assoc 2002;133:323-9.

5. Manolagas SC. Birth and death of bone cells: basic regulatory mechanisms and implications for the pathogenesis and treatment of osteoporosis. Endocr Rev 2000;21:115-37.

6. Duarte PM, Gonçalves P, Casati MZ, de Toledo S, Sallum EA, Nociti FH Jr. Estrogen and alendronate therapies may prevent the influence of estrogen deficiency on the tooth-supporting alveolar bone: a histometric study in rats. J Periodontal Res 2006;41:541-6.

7. Esfahanian V, Shamami MS, Shamami MS. Relationship between osteoporosis and periodontal disease: review of the literature. J Dent (Tehran) 2012 Fall;9:256-64.
8. Güncü GN, Tözüm TF, Caglayan F. Effects of endogenous sex hormones on the periodontium-review of literature. Aust Dent J 2005;50:138-45.

9. Preshaw PM. Oral contraceptives and the periodontium. Periodontol 2000. 2013;61:125-59.

10. Bhardwaj A, Bhardwaj SV. Effect of menopause on women's periodontium. J Midlife Health 2012;3:5-9.

11. Yildirim T, Kaya F. The Effects of Menopause on Periodontal Tissue. International Dental Research@ 201 1, vol. $1, n^{\circ} 3.2$

12. Machtei EE, Mahler D, Sanduri H, Peled M. The effect of menstrual cycle on periodontal health. J Periodontol 2004;75:408-12.

13. Pizzo G, Guiglia R, Licata ME, Pizzo I, Davis JM, Giuliana G. Effect of hormone replacement therapy (HRT) on periodontal status of postmenopausal women. Med Sci Monit 201 1;17:PH23-7.

14. Saraiva, Patrícia Pinto; Múfalo, Paula Simões; Moraes, Lúcia Abreu de; Padovani, Carlos Roberto. Influence of the estrogen in the periodontium of menopausal women. Salusvita 2007;26:95-104. Tab.

15. Santos, Juliana Franco dos; Pillon, Flávio de Lecue. The influence of female sexual hormones on clinical manifestation of periodontal diseases - Review. Periodontia 2009; 19:34-40.

16. Jeffcoat M. The association between osteoporosis and oral bone loss. J Periodontol 2005;76:2125-32.

\section{CORRESPONDENCIA}

Victor Fabrizio Cabrera Pazmino

Universidade Estadual Paulista "Júlio de Mesquita

Filho"

Faculdade de Odontologia de Araçatuba

Departamento de Cirurgia e Clínica Integrada

Disciplina Periodontia/UNESP

Rua José Bonifácio 11-93

Bairro Jardim Paulista. CEP 16015050

Araçatuba - São Paulo. Brasil

Correos electrónicos: drvifacapa@hotmail.com drfabriziocabrera@hotmail.com 